\title{
Apreçamento das Opções de Venda sobre Contratos Futuros de Café Arábica com a Fórmula de Black: uma análise exploratória
}

\author{
Julyerme Matheus Tonin ${ }^{1}$
}

Alexandre Bragança Coelho²

\begin{abstract}
Resumo: O expressivo aumento dos derivativos no período recente, especialmente o mercado de opções, faz desse instrumento um forte candidato para suprir a necessidade de redução do risco de preço no setor cafeeiro. Assim, buscou-se analisar a aplicabilidade da Fórmula de Black no apreçamento de opções sobre futuros de café arábica. Diferente de outros trabalhos sobre o tema buscou-se incorporar na análise diversos extratos da amostra utilizada: análise dos resultados em diferentes anos, em diferentes vencimentos, com diferentes métodos de extração da volatilidade, com diferentes graus de moneyness e maturidade. Os resultados mostram o melhor desempenho dos modelos de apreçamento das opções com a utilização de volatilidade implícita ponderada, sendo que, identifica-se a presença do efeito smile nessa volatilidade. De modo geral, há uma subavaliação dos prêmios das opções com o uso da volatilidade histórica e superavaliação com o uso da volatilidade implícita e as opções com período médio de volatilidade são as que apresentam os melhores resultados de apreçamento das opções.
\end{abstract}

Palavras-chaves: Apreçamento de Opções, Modelo Black e Scholes, Volatilidade, Café Arábica

JEL: G20, G32, Q14 


\begin{abstract}
The expressive increase of derivatives in the recent period, especially the options market, makes this instrument a strong candidate to supply the need for price risk reduction in the coffee industry. Therefore, it aimed to analyzed applicability of the Black's Formula for options pricing on arabica coffee futures. Differently other papers on the theme was looked to include in the analysis several extracts of the sample: analysis of results in different years, in different expirations, with different methods of volatility extraction, with different moneyness degrees and maturity. The results show the best performance of the options pricing models in which it used the weighted implied volatility, and identifies the presence of smile effect in this volatility. In general, there is an undervaluation of the option with the use the historical volatility and overvaluation of the options using the implied volatility and the options with medium period of volatility are the ones that present the best pricing results.
\end{abstract}

Keywords: Option Pricing, Black and Scholes Model, Volatility, Coffee Arabica

\title{
Introdução
}

A cultura do café, além da reconhecida importância histórica, destaca-se como atividade de alto valor econômico e social. No contexto histórico, o "ciclo do café" contribuiu com parte substancial da geração de superávits comerciais que propiciaram o início da industrialização brasileira, deixando de herança amplos mercados interno e externo para essa commodity e um parque produtivo complexo e diverso (FARINA e ZYLBERSZTAJN, 1998).

Com a ausência da intervenção estatal da atividade a partir da década de 1990, com a extinção do Instituto Brasileiro do Café e dos Acordos Internacionais do Café, a redução de riscos se tornou imperativa, ampliando assim a importância das políticas privadas de gestão de risco, especialmente os derivativos agropecuários. Nesse contexto, a Future Industry Association (FIA, 2011) destaca o expressivo volume de opções em 2010, com 11,12 bilhões de contratos negociados, a nível mundial.

O fortalecimento institucional da Bolsa de Mercadorias e Futuros (BM\&F), com a internacionalização dos mercados futuros agropecuários, em dezembro de 1999, contribuiu para a disseminação dos derivativos agropecuários, propiciando a participação de não-residentes. Outro passo importante foi dado em março de 2008 com a fusão da BM\&F e a Bolsa de Valores de São Paulo (BOVESPA), minorando os custos operacionais (BMFBOVESPA, 2010).

Apesar do expressivo volume de contratos de opções negociados no mercado internacional, do fortalecimento institucional da bolsa brasileira e do ambiente de estabilidade no mercado interno favorável a atuação nesses mercados, a 
liquidez dos contratos de opção na BM\&FBOVESPA ainda é baixa. De acordo com BM\&FBOVESPA (2011), em 2010 os contratos agropecuários representaram apenas $0,43 \%$ dos contratos negociados na bolsa, sendo que, desse montante, 24,02\% referem-se a contratos futuros e opções de café arábica.

Em face das mudanças institucionais vivenciadas no setor cafeeiro e a crescente importância do mercado de opções na gestão de risco, torna-se necessário uma análise mais detalhada sobre a determinação do prêmio das opções. Nesse sentido, busca-se avaliar a aplicabilidade da fórmula de Black no apreçamento de opções de venda sobre futuros de café arábica. Optouse pela análise das opções de venda, pois as mesmas são utilizadas, com frequência por produtores rurais e demais hedgers para redução dos riscos associados a queda nos preços. Para esse fim, utilizam-se diferentes métodos de extração da volatilidade histórica e implícita, evidenciando os resultados em diferentes extratos da amostra: diferentes períodos para o cálculo da volatilidade histórica; grau de moneyness; diferentes vencimentos; diferentes anos e diferentes maturidades.

Diferente dos trabalhos já publicados sobre o tema, como Leite (2000), Ramos da Silva e Macedo (2003) e Coelho et al. (2009), o presente estudo busca realizar uma análise exploratória para evidenciar as circunstâncias em que a Fórmula de Black pode ser aplicada e qual a influência da volatilidade e do grau de maturidade nos resultados de apreçamento de opções sobre futuro de café arábica.

\section{REFERENCIAL TEÓRICO}

\subsection{Abordagem de Black e Scholes: A fórmula de Black}

Fisher Black e Myers Scholes desenvolveram em 1973 um modelo para apreçamento de opções. Hull (2006) destaca que esses autores utilizaram uma fórmula que considera o tempo contínuo na análise, para o cálculo do valor teórico de uma opção de compra do tipo européia, exercida sobre um objeto que não paga nenhum dividendo e é livremente negociada no mercado. Para Leuthold et al. (1989) o modelo original de Black e Scholes, sofreu algumas adaptações para ser empregado em opções sobre futuros, dando origem a fórmula de Black (1976). Porém as premissas básicas do modelo não sofreram alteração:

- O comportamento do preço do ativo segue uma distribuição lognormal, com volatilidades dos retornos diários constantes;

- Ativos perfeitamente divisíveis, sem custos operacionais e impos- 
tos;

- $\quad$ O ativo objeto não receberá dividendos durante a vida da opção;

- Não há oportunidades de arbitragem sem risco;

- $\quad$ Os empréstimos são realizados à taxa de juros livre de risco.

A dinâmica dos preços ou retornos do ativo-objeto é representada por um modelo estocástico simples de movimentos de preços, o passeio aleatório. Segundo Hull (2006) esse processo, também denominado movimento browniano, é útil no modelo de apreçamento, pois assume o pressuposto de que o mercado reage imediatamente ao surgimento de uma nova informação, sendo que a dinâmica do retorno do ativo pode ser representada por:

$$
\$_{t} / S_{t}=\mathrm{md}+\mathrm{s} \mathbb{Z}{ }_{t}
$$

em que, $\$_{t} / S_{t}$ é a taxa discreta de retorno do ativo; $\boldsymbol{t}$ é o intervalo de tempo infinitesimal; mé o valor esperado instantâneo da taxa de retorno composta; $\mathrm{s}$ representa a volatilidade, e $\mathbb{Z}{ }_{t}$ variável aleatória com distribuição normal.

Com a aplicação do cálculo integral, algumas noções de probabilidade e demais considerações que fogem do escopo deste trabalho ${ }^{3}$, as fórmulas de Black e Scholes para calls e puts européias podem ser simplificadas para:

$$
c=e^{-T}\left[\begin{array}{lll}
\mathbb{N} & \left(d_{1}\right)-X & \left.\left(d_{2}\right)\right]
\end{array}\right.
$$

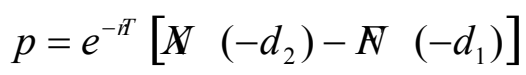

em que,

$$
\begin{array}{r}
d_{1}=\frac{\mathbf{h}\left(\frac{K}{S}\right)+\left(b+\frac{\mathrm{s}^{2}}{2}\right)(T-t)}{\mathrm{s} \sqrt{(T-t)}} \mathrm{e} \\
d_{2}=\frac{\mathbf{h}\left(\frac{K}{S}\right)+\left(b-\frac{\mathrm{s}^{2}}{2}\right)(T-t)}{\mathrm{s} \sqrt{(T-t)}}
\end{array}
$$

dado que,

$c$ e $p$ são o preço de uma call e da put européia, respectivamente;

$F$ e $K$ são preço do contrato futuro e o preço de exercício da opção, respectivamente;

3 A resolução da equação diferencial parcial de Black-Scholes pode ser encontrada em Luccas (2007). 
$N(x)$ é a função de densidade de probabilidade acumulada (normal padrão).

$b$ é o custo de carregamento e $\mathbf{S}$ é a volatilidade.

As equações (1.2) e (1.3) são considerados na literatura como o modelo de Black e Scholes Generalizado aplicado para as opções de compra e venda, respectivamente. Haug (1997) destaca que essa generalização deve-se a variável $b$, que pode ser considerada de diferentes formas no modelo: $b=r$ corresponde ao modelo original de Black e Scholes (1973) e $b=0$ equivale a fórmula de Black (1976) para precificar opções sobre contratos futuros, o enfoque desse trabalho.

\section{METODOLOGIA}

\subsection{Operacionalização das Variáveis}

No presente estudo, para o cálculo da volatilidade histórica é necessário construir uma série de logaritmo de preço do ativo subjacente. O procedimento mais empregado é a formação de uma série única de preços futuros composta pelas cotações do primeiro vencimento em aberto. Porém, Sanvicente e Monteiro (2005) destacam que a pressão decorrente da proximidade do vencimento dos contratos futuros (expiration-day effect), causa um volume de negociação e volatilidade acima do normal.

No caso do café, a menor liquidez em alguns vencimentos (especialmente o vencimento de julho), causa a falsa impressão de queda na volatilidade. Para contornar esses problemas, o primeiro vencimento em aberto é sempre substituído, quando o próximo vencimento apresentar um maior volume de contratos negociados por pelo menos 5 dias úteis seguidos. Essa técnica considera a rolagem de posições ${ }^{4}$ entre os diferentes vencimentos, e garante que a série de preços do ativo subjacente reflita o maior volume de contratos negociados possíveis, sendo útil para analisar a atuação dos agentes.

O tempo de maturidade das opções foi calculado como uma proporção dos dias úteis no ano (T-t/252). No presente trabalho, conforme o proposto por Malz (2000), foram retiradas da análise as opções muito próximas ao vencimento, opções com tempo de maturidade de até 5 dias ${ }^{5}$, dada a baixa liquidez dessas . Nesse contexto, também exclui-se as opções seriais que são lançadas em meses em que não há um contrato futuro vincendo, devido à pequena

4 Rolagem de posição é a compra/venda de contratos futuros que estão sendo transacionados em um determinados momento (com maior liquidez) e substituir esses contratos por novas posições a medida que estes contratos vão se aproximando do vencimento.

5 A escolha do número é arbitrária, correspondendo em dias úteis a uma semana de negociação. 
quantidade de negócios efetuados.

A taxa de juros considerada é a taxa de juros efetiva anual continuamente capitalizada, dada por $r=\ln (1+i)$ em que $i$ representa a taxa de juros livre de risco. Quanto ao preço de exercício, calculou-se o índice de moneyness, que para Ederigton e Guan (2000) é um índice que representa o quão dentrodo-dinheiro está a opção em questão, sendo que a forma analítica pode ser descrita por:

$$
\mathbb{N}=X_{t} \cdot e^{-T} / S_{t}
$$

em que, $S_{t}$ é o preço do ativo-subjacente; $X_{t}$ o preço de exercício; $r$ a taxa de juros livre de risco e $T$ o tempo de maturidade da opção.

Após a elaboração do índice, adotou-se a seguinte classificação: uma opção é considerada dentro-do-dinheiro ou in-the-money (ITM) se o resultado da equação (3.1) for $M I>1, \mathbb{\theta}$; a opção é classificada como fora-do-dinheiro ou out-of-the-money (OTM) se o índice for $M<0,9$ e para os demais casos é classificada como opção no-dinheiro ou at-the-money (ATM) com $0,9 \leq M \leq 1,0$.

Esse mesmo critério de classificação foi adotado por Vitiello Jr (2000), Mikoszewski (2003) e Luccas (2007). Com base no proposto por Hauser e Lauterbach (1997), foram criadas outras duas categorias, as opções muito dentro-do-dinheiro ou deep in-the-money (DITM) e muito fora-do-dinheiro ou deep out-of-the-money (DOTM), opções com dois desvios-padrão abaixo ou acima de uma opção plenamente no-dinheiro $(M=1)$, respectivamente.

Essa categorização possibilita analisar as opções de acordo com a proximidade do dinheiro, mas não inibe a ocorrência de outliers. Seguindo a recomendação de Bustos (1989), o procedimento de detecção dos outliers foi feito por meio do teste de Grubbs ${ }^{6}$.

\subsection{Cálculo da Volatilidade Histórica}

Segundo Luccas (2007), pode-se entender a volatilidade histórica como o desvio-padrão da mudança no logaritmo natural do preço do ativo-objeto que é esperado ao longo do prazo de vida da opção, expressa em porcentagem ao ano. Como utiliza-se uma série de logaritmo de preço do ativo subjacente para o cálculo da volatilidade, é necessário testar a normalidade dos resíduos (teste de Jarque-Bera ${ }^{7}$ ).

\footnotetext{
Para maiores detalhes, ver Bustos (1989).

A estatística do teste é $\mathbb{B}=n \cdot\left[A^{2} / 6+(C-3)^{2} / \mathbb{Z}\right]$ em que A representa a assimetria e C a curto-
} 
Segundo Purcell e Koontz (1999), o cálculo da volatilidade por indução retroativa, a partir da série temporal histórica dos preços diários do fechamento do ativo-objeto, pode ser obtido com a aplicação da fórmula:

$$
\text { (4.1) } s=\sqrt{\frac{1}{n-1} \sum_{i=1}^{n}\left(r_{i}-\bar{r}\right)^{2}} \quad \text { e } \mathrm{s}=s \sqrt{252}
$$

em que, $s$ é a volatilidade histórica; $n-1$ é o número de variações nos preços; $r_{i}=\ln \left(S_{i} / S_{i-1}\right)$, sendo $S_{i}$ o preço do ativo no final do i-ésimo período, com média $\bar{r}$, e $\mathrm{S}$ é a volatilidade em termos anualizados.

A determinação de $n$ não é trivial, pois o acréscimo de muitas observações de fechamento de preço do ativo objeto conduz a uma exatidão maior, mas dados muito antigos podem não ser relevantes para prever o futuro. Convencionalmente, empregam-se médias móveis de forma recursiva para calcular a volatilidade histórica, considerando um tamanho da amostra predefinido. Como não há um critério a priori que define o tamanho da amostra, no presente trabalhos testou-se as seguintes janelas recursivas: 30, 45, 60, 75 e 90 dias.

Para Gentil Junior (2007), os resultados observados para a volatilidade com diferentes janelas temporais não são isentos de críticas, dado que a base de dados é composta exclusivamente de preços de fechamento do ativo objeto. Para captar a volatilidade intradiária, ou seja, a volatilidade no decorrer dos dias de negociação, Parkinson (1980) elaborou a seguinte fórmula:

$$
\mathrm{s}=\frac{1}{2 n \sqrt{\ln (2)}} \sum_{i=1}^{n} \mathrm{~h}\left(\frac{\operatorname{High}_{i}}{\text { Low }_{i}}\right)
$$

em que, $H i g h_{i}$ e $L o w_{i}$ referem-se as cotações mais alta e a mais baixa do preço do ativo objeto, respectivamente, em um determinado dia de negociação.

Nesse contexto, Garman e Klass (1980) desenvolveram um cálculo de volatilidade que pudesse captar a volatilidade intradiária sem desconsiderar a volatilidade entre os dias de negociação. 
$\mathrm{S}=\sqrt{\frac{1}{n} \sum_{i=1}^{n}\left[\mathrm{~h}\left(\frac{\text { High }_{i}}{\text { Low }_{i}}\right)\right]^{2}-\frac{1}{n} \sum_{i=1}^{n}[2 \ln (2)-1] *\left[\mathrm{~h}\left(\frac{\text { Close }_{i}}{\text { Close }_{i-1}}\right)\right]^{2}}$ em que, Close $e_{i}$ é o fechamento do pregão atual e $C l o s e_{i-1}$ o fechamento do pregão do dia anterior.

Diversas técnicas de modelagem da volatilidade estocástica também foram desenvolvidas, além da utilização da volatilidade histórica. Especificamente, utilizou-se o modelo GARCH (General Autoregressive Conditional Heterocedasticity):

$$
\mathrm{S}_{t}^{2}=\mathrm{W}+\mathbf{a} u_{t-i}^{2}+\mathbf{b}{ }_{t-i}^{2}
$$

em que $\mathrm{W}$ refere-se a um termo constante; a consiste em uma estimativa similar ao modelo ARCH e b é a previsão da volatilidade feita no período $t-i\left(\mathrm{~s}_{t-i}^{2}\right)$.

Para efeito de comparação com as demais metodologias de cálculo da volatilidade histórica, utiliza-se a mesma forma de cálculo empregado por Aguilar (1999). Para o cálculo da volatilidade do primeiro dia do período analisado, define-se uma janela de estimação a priori $(30,60 \text { e } 90 \text { dias })^{8}$. No próximo passo, a janela de estimação para os parâmetros do modelo GARCH é aumentada em uma observação, sendo que a observação inicial é excluída da análise. Esse procedimento será realizado até que se obtenham previsões de volatilidade para todas as observações da série analisada.

Enfim, no presente trabalho, foram empregadas as seguintes volatilidades históricas: média móvel (VMA), procedimento High-Low de Parkinson (VHL), procedimento Close-High-Low de Garman e Klass (VCH) e o modelo GARCH $(1,1)$.

\subsection{Cálculo da Volatilidade Implícita}

Para Gabe e Portugal (2004), a volatilidade implícita está associada ao preço da opção, sendo que a utilização da volatilidade implícita é uma forma de avaliar as expectativas dos agentes econômicos quanto a volatilidade futura. O cálculo da volatilidade implícita é realizado a partir do prêmio observado da opção, por meio da aproximação de Newton-Rapson ${ }^{9}$, que segundo Stoll

8 Dada ao maior custo computacional para o cálculo da volatilidade com o modelo GARCH (1,1), as janelas de 45 e 75 foram desconsideradas da análise.

9 Algoritmo muito utilizado na precificação de opções, que pode ser calculado em planilhas do Excel, por meio de macro do Visual Basic. 
e Whaley (1993) pode ser descrita da seguinte forma:

$$
\mathbf{S}_{i+1}=\frac{\mathbf{s}_{i}-c\left(\mathbf{S}_{i}\right)-c_{m}}{\partial c / \partial \mathbf{s}_{i}}
$$

em que, $c\left(\mathbf{s}_{i}\right)$ é o preço observado e $c_{m}$ o preço calculado da opção; $\partial c / \partial \mathbf{s}_{i}$ é a medida de sensibilidade do valor da opção em relação a volatilidade (letra grega vega ${ }^{10}$ ).

Cabe destacar que, no método de Newton-Rapson, é necessário conhecer a medida de sensibilidade vega, medida essa que não é prontamente observável em opções exóticas e algumas opções americanas. Resolvendo a equação (5.1) obtêm-se a volatilidade implícita imputada no modelo de apreçamento. A definição $S_{i+1}$ demonstra que essa volatilidade não é uma variável contemporânea, ou seja, a volatilidade implícita obtida com os dados de um determinado dia será utilizada como estimativa da volatilidade futura para o cálculo do preço teórico da opção no dia seguinte.

No tocante ao café arábica na BM\&FBOVESPA, em muitos casos, a volatilidade implícita calculada não pode ser utilizada para estimação no dia seguinte, por não haver negociações com o ativo subjacente com as mesmas características (mesma série de opções, mesmo vencimento, etc.) do ativo que foi utilizado para calcular previamente a volatilidade implícita. Para contornar esse problema, essa volatilidade é ponderada pela média das volatilidades implícitas dos ativos subjacentes com as mesmas características

$$
\hat{\mathrm{s}}_{\text {ponderado }}=\mid \hat{\mathrm{s}}_{i} \operatorname{com} \mathrm{I}=1-\left(\hat{\mathrm{s}}_{i}-\overline{\mathrm{s}}\right)
$$

em que, I é um peso que desconta o desvio da volatilidade implícita em relação a média das volatilidades implícitas para o mesmo vencimento $(\overline{\mathrm{S}})$.

Em outras situações, há mais de uma opção negociada para um mesmo contrato futuro e geralmente os valores das volatilidades implícitas são diferentes entre essas opções. Para Luccas (2007), esse é um fato estilizado no apreçamento de opções, conhecido como sorriso da volatilidade. Para mitigar os problemas associados com o uso da volatilidade implícita simples, foram desenvolvidos alguns métodos para ponderar a volatilidade, levando em conta a distância em relação ao dinheiro. Neste trabalho, será utilizado o procedimento de Macbeth e Merville (1979). Segundo o autor, a volatilidade implícita de uma opção no-dinheiro é boa referência para a volatilidade do ativo objeto e, como cada série de opções lançadas sobre o mesmo contrato futuro pode fornecer diferentes resultados, é necessário estimar a regressão apresentada na equação (5.3) para se obter uma estimativa da volatilidade do contrato futuro:

10 O vega de uma opção de compra, por exemplo, é dado por $\mathrm{n}_{C}=\mathrm{n}_{C}(t, S, \mathrm{~s})=\partial C / \partial \mathrm{s}$ e demonstra quanto o preço da opção é afetado por mudanças na volatilidade. 
(5.3)

$\mathrm{S}_{i j t}=\mathrm{S}_{i 0 t}+\mathrm{S}_{i 1 t} m_{i j t}+\mathrm{e}_{j t}$

em que, $S_{i j t}$ é a volatilidade implícita da opção $j$ sobre o contato futuro $i$ no instante $t ; \mathbf{S}_{i 0 t}$ é a volatilidade implícita de uma opção no-dinheiro; $\mathbf{S}_{i 1 t}$ é um parâmetro da regressão; $m_{i j t}=\left(F_{i}-X_{j}\right) / X_{j}, \operatorname{com} F_{i t}$, o preço do contato futuro e $X_{i j}$ o preço de exercício da opção, e e ${ }_{j t}$ é o erro padrão.

De forma simplicada, Vitiello Jr (2000) destaca que o processo para obtenção da volatilidade implícita de uma opção no-dinheiro segue as seguintes etapas:

Calcular a volatilidade implícita, $\mathrm{s}_{i j t}$, ou seja, calculam-se, no dia $t$, as volatilidades implícitas das opções lançadas sobre determinado contrato futuro $i$;

Tendo-se calculado $\mathbf{S}_{i j t}$, utiliza-se a equação (5.3) para se obter $\mathbf{S}_{i 0 t}$, a volatilidade implícita no-dinheiro do contrato futuro $i$, no instante $t$.

\subsection{Método de Avaliação dos Modelos de Apreçamento}

Para avaliar a capacidade preditiva dos modelos, apurou-se o erro relativo médio, para verificar se os modelos de apreçamento subavaliam ou superavaliam o valor do prêmio pago pela opção, dado por:

(6.1)

$$
E R M=\sum \frac{\left|c_{i}-\hat{c}_{i}\right|}{c_{i}}
$$

em que, $c_{i}$ e $\hat{c}_{i}$ são o preço de mercado e o preço estimado da opção.

Como cada observação refere-se a um negócio efetuado, em que são negociadas lotes de opções de venda, com o mesmo prêmio, os resultados obtidos para o ERM foram ponderados pelo número de contratos. Essa medida reduz o impacto negativo de negócios efetuados com poucas opções e com elevados erros de apreçamento nos resultados obtidos.

Quanto a metodologia de análise estatística, cabe destacar que os dados foram submetidos à análise de Variância (ANOVA), seguidos do teste de Tukey. O objetivo da ANOVA é avaliar se as diferenças observadas entre as médias das amostras são estatisticamente significativas. O teste de Tukey, segundo Hoffmann (2006), consiste em comparar médias duas a duas, com amostras de tamanhos iguais, buscando identificar um valor de diferença mínima significativa e compará-lo com valores críticos "estudentizados”. A diferença mínima significativa (DMS) é dada por: 
(6.2)

$$
\Delta_{0}=q_{0} \sqrt{s^{2} / n}
$$

em que, $s^{2}$ é a soma do quadrado dos resíduos e $q_{0}$ é o valor crítico da amplitude total "estudentizada" que depende do nível de significância adotado e $n$ é o número de observações ( $n_{i}$ e $n_{j}$ representam grupos de tamanhos diferentes).

A seguir, comparam-se os valores absolutos das estimativas dos contrastes $u_{i}-u_{j}$ dados por $\left|m_{i}-m_{j}\right|$, como o valor de $\Delta_{0}$. Se o valor de $\left|m_{i}-m_{j}\right| \geq \Delta_{0}$ rejeita-se ao nível de significância adotado a hipótese $H_{0}$ de que todas as diferenças de pares de média sejam nulas.

\subsection{Fonte de Dados}

Com o intuito de atender a análise de apreçamento coletou-se no Sistema de Recuperação de Informações da BM\&FBOVESPA, o resumo estatístico do pregão referente ao período de 01 de janeiro de 2003 a 31 de dezembro de 2009, contendo as seguintes informações: ajuste diário do contrato futuro de café arábica (ativo subjacente), maior cotação e menor cotação negociada, séries de opções sobre contratos futuros de café arábica autorizadas com respectivos prêmios pagos, preço de exercício, dias úteis até o vencimento e número de contratos negociados (BVMF, 2010).

Dado que a BM\&FBOVESPA apresenta em seu relatório as séries de opções de compra e de venda negociadas e o número de negócios efetuados ${ }^{11}$, no período de análise a amostra utilizada compreende 949 negócios efetuados com opções de venda. No presente estudo utilizou-se as opções de venda, com maior liquidez, dada que as mesma são utilizadas na gestão do risco de preço, especificamente contra possíveis quedas de preços, sendo úteis aos produtores rurais e demais hedgers. Como em cada "negócio efetuado" são transacionadas diversas opções, a amostra utilizada representa 105.655 opções de venda. Seguindo o proposto por Gabe e Portugal (2004) e Silva e Guimarães (1999), adotou-se como proxy a taxa de juros dos Certificados de Depósitos Interbancários (CDI) anual e composta, obtida junto ao Banco Central (BC, 2010). Os dados foram operacionalizados por meio do software Eviews 6.0.

11 Negócio efetuado é a forma de contabilizar a participação de cada agente no mercado de opções. Por exemplo, se só um agente negociar 50 opções em um determinado dia, nessa data é contabilizado um negócio efetuado e 50 contratos negociados. 


\section{Resultados e discussão}

\subsection{Análise preliminar dos dados}

A Figura 1 demonstra o comportamento de preços do ativo subjacente, ou seja, o contrato futuro de café arábica. É possível identificar facilmente o padrão sazonal de variação dos preços no decorrer de cada ano, bem como o ciclo de alta desse produto. Para o Instituto de Economia Agrícola (IEA), 2002 delimita um período de acentuada queda nos preços do café, sendo que após uma lenta recuperação (2003 e 2004) inicia-se um novo ciclo plurianual de elevação nos preços do café. A série de retornos ressalta a variação dos preços, sendo que, a inspeção visual permite apenas detectar o padrão inconstante das oscilações de preços.

FIGURA 1 - COMPORTAMENTO DOS PREÇOS FUTURO E DO RETORNO DOS PREÇOS FUTUROS DE CAFÉ ARÁBICA, NO PERÍODO DE 2003 A 2009.

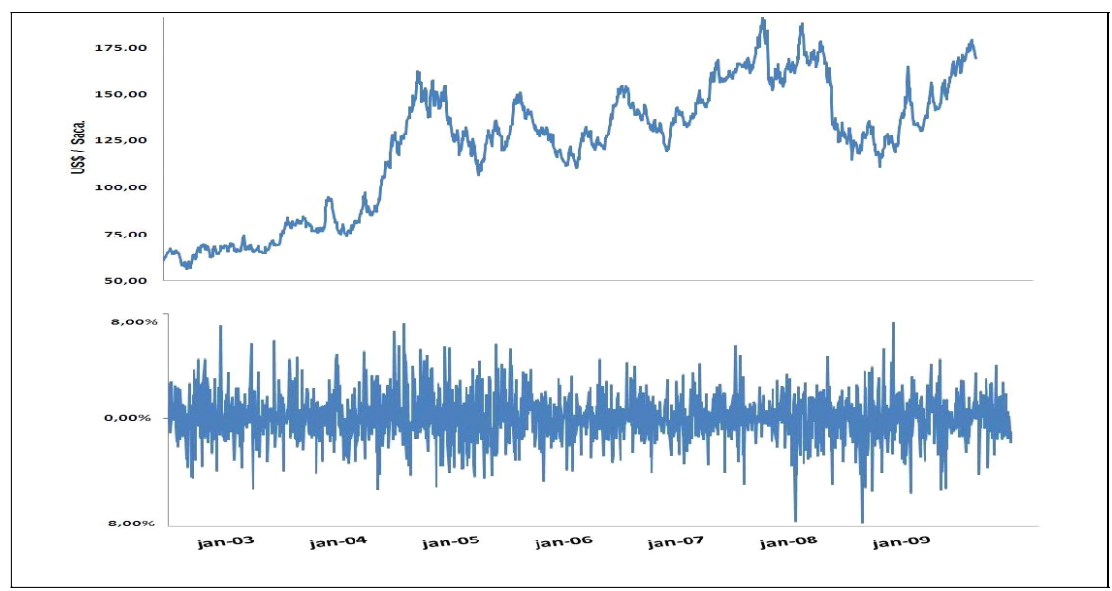

FONTE: BM\&FBOVESPA (2010)

As características observadas na série de retorno são compatíveis com a hipótese de não-normalidade e leptocurtose, ou seja, um choque que afete o preço futuro do café arábica pode implicar em vários períodos de volatilidade no mercado, fato esse que pode ser comprovado por meio do teste de normalidade de Jarque-Bera (Figura 2). 
FIGURA 2 - HISTOGRAMA E SUMÁRIO ESTATÍSTICO DOS RETORNOS SOBRE OS PREÇOS FUTUROS DE CAFÉ ARÁBICA NA BM\&FBOVESPA, NO PERÍODO DE 2003 A 2009.

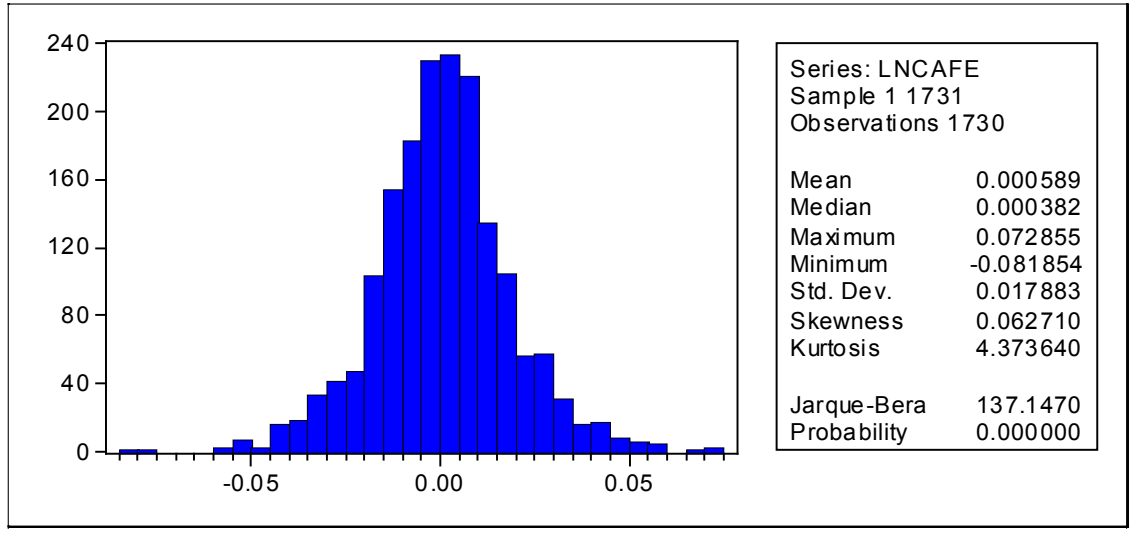

Fonte: Dados da Pesquisa

No tocante ao cálculo da volatilidade histórica, buscou-se incorporar na análise da série de retornos diários por meio do emprego de média móvel, a análise da volatilidade no decorrer dos dias de negociação, com o procedimento de Parkinson (1980) e o procedimento de Garman e Klass (1980) e a volatilidade condicional, com a estimação do modelo GARCH $(1,1)^{12}$. Na Figura 2 são apresentados os cálculos dos diferentes métodos de extração da volatilidade, sendo que, para efeito de comparação, utiliza-se uma janela recursiva de 30 dias. Verifica-se uma semelhança entre as volatilidades VMA, VHL e VHLC. O procedimento de Parkinson (1980) capta a volatilidade intradiária, por meio da análise dos preços mínimos e máximos diários, enfatizando assim, os períodos de baixa volatilidade no mercado. O método GARCH destoa das demais volatilidades históricas, captando picos de volatilidade, fato esse que demonstra a dependência temporal nos retornos diários dos ativos (Figura 3). 
FIGURA 3 - VOLATILIDADES HISTÓRICAS ANUALIZADAS DO RETORNO DOS PREÇOS FUTUROS DE CAFÉ ARÁBICA NA BM\&FBOVESPA.

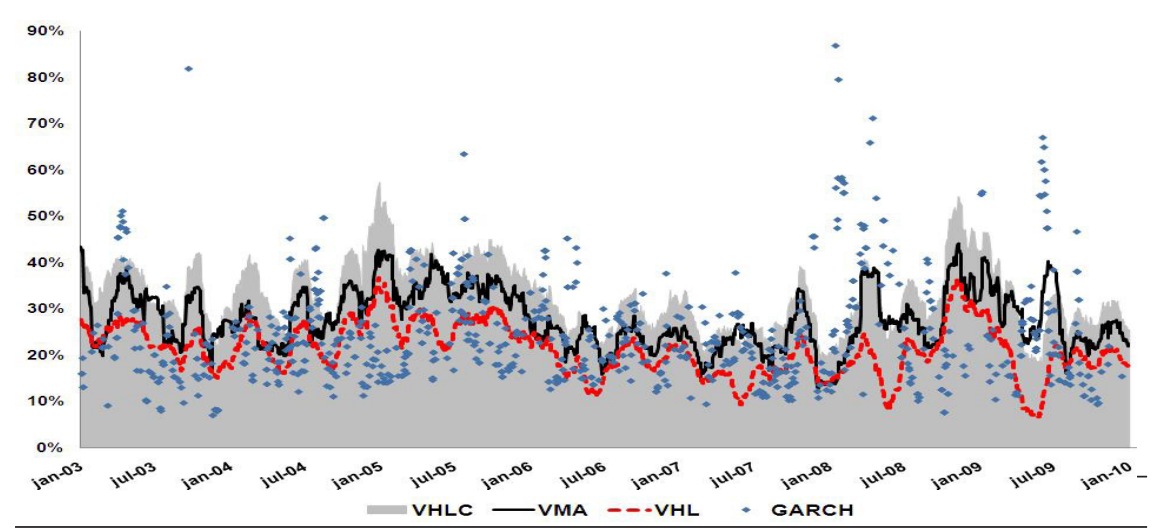

FONTE: Dados da Pesquisa. ${ }^{*}$ ) Volatilidades: média móvel (VMA); procedimento High-Low de Parkinson (VHL) ; procedimento High-Low-Close de Garman e Klass (VHLC) e o modelo GARCH $(1,1)$.

Feito o cálculo da volatilidade pelos diferentes métodos de indução retroativa, parte-se para o cálculo da volatilidade implícita. Obedecendo aos critérios de Macbeth e Merville (1979), procedeu-se ao cálculo da volatilidade implícita no-dinheiro, doravante denominada VOI e a volatilidade implícita ponderada pela média das volatilidades do mesmo vencimento, doravante denominada VOI-P. Na Figura 4, verifica-se que a ponderação reduziu os efeitos de picos de volatilidade. 


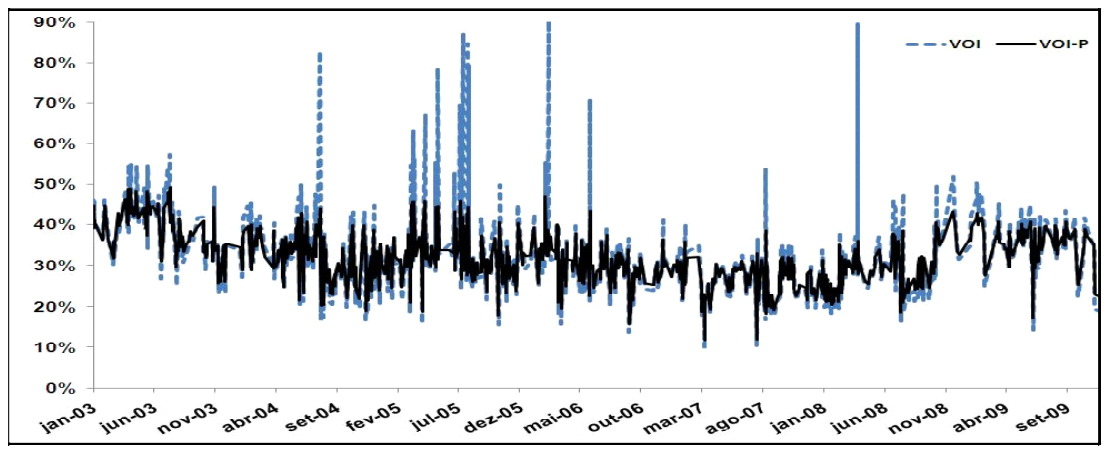

FONTE: Dados da Pesquisa. ${ }^{*}$ ) As volatilidade são: volatilidade implícita no-dinheiro (VOI) calculada com base no procedimento de Macbeth e Merville (1979) e volatilidade implícita no-dinheiro ponderada (VOI-P).

\subsection{Resultados da Apreçamento das Opções de Venda}

Para avaliar as 949 observações, representativas de 105.655 opções de venda sobre futuros de café arábica na BM\&FBOVESPA, são comparados os preços teóricos com os preços de mercado (Tabela 1). 
TONIN, M. T. ; COELHO, A. B. Apreçamento sobre as opções de venda ...

TABELA 1 - RESULTADOS DA APREÇAMENTO DAS OPÇÕES DE VENDA COM VOLATILIDADES HISTÓRICA E IMPLÍCITA PARA DIFERENTES JANELAS TEMPORAIS

\begin{tabular}{|c|c|c|c|c|c|c|c|}
\hline & & ERM & $\begin{array}{c}\text { Média } \\
\text { Modelo }\end{array}$ & $\begin{array}{c}\text { Diferença do } \\
\text { Mercado }\end{array}$ & $\begin{array}{c}\% \text { Sub } \\
\text { Precificado }\end{array}$ & $\begin{array}{c}\text { Erro } \\
\text { Mínimo }\end{array}$ & $\begin{array}{c}\text { Erro } \\
\text { Máximo }\end{array}$ \\
\hline \multirow{5}{*}{$\sum_{>}^{\mathbb{3}}$} & 30 & $-15,71 \%$ & 5,06 & $0,92^{\mathrm{NS}}$ & $70,81 \%$ & $-99,99 \%$ & $368,42 \%$ \\
\hline & 45 & $-15,14 \%$ & 5,05 & $0,92^{\mathrm{NS}}$ & $71,55 \%$ & $-99,93 \%$ & $356,99 \%$ \\
\hline & 60 & $-13,65 \%$ & 5,06 & $0,91^{\mathrm{NS}}$ & $69,34 \%$ & $-98,83 \%$ & $359,86 \%$ \\
\hline & 75 & $-12,31 \%$ & 5,14 & $0,83^{\mathrm{NS}}$ & $68,18 \%$ & $-98,98 \%$ & $342,83 \%$ \\
\hline & 90 & $-11,39 \%$ & 5,19 & $0,79^{*}$ & $67,76 \%$ & $-98,91 \%$ & $360,15 \%$ \\
\hline \multirow{5}{*}{$\mathbb{7}$} & 30 & $-47,50 \%$ & 3,73 & $2,24^{\mathrm{NS}}$ & $91,25 \%$ & $-100,00 \%$ & $324,99 \%$ \\
\hline & 45 & $-50,21 \%$ & 3,70 & $2,27^{\mathrm{NS}}$ & $92,41 \%$ & $-100,00 \%$ & $317,07 \%$ \\
\hline & 60 & $-52,28 \%$ & 3,62 & $2,35^{\mathrm{NS}}$ & $94,20 \%$ & $-100,00 \%$ & $314,17 \%$ \\
\hline & 75 & $-52,28 \%$ & 3,62 & $2,35^{\mathrm{NS}}$ & $94,20 \%$ & $-100,00 \%$ & $314,17 \%$ \\
\hline & 90 & $-50,31 \%$ & 3,69 & $2,28^{\mathrm{NS}}$ & $94,10 \%$ & $-100,00 \%$ & $324,45 \%$ \\
\hline \multirow{5}{*}{$\frac{\pi}{2}$} & 30 & $26,27 \%$ & 6,06 & 0,68 & $50,79 \%$ & $-98,95 \%$ & $404,52 \%$ \\
\hline & 45 & $20,97 \%$ & 6,04 & 0,42 & $49,53 \%$ & $-97,97 \%$ & $433,42 \%$ \\
\hline & 60 & $22,89 \%$ & 6,06 & 0,28 & $48,47 \%$ & $-96,83 \%$ & $384,60 \%$ \\
\hline & 75 & $21,28 \%$ & 5,99 & 0,23 & $45,84 \%$ & $-97,76 \%$ & $448,99 \%$ \\
\hline & 90 & $23,98 \%$ & 5,98 & 0,24 & $43,73 \%$ & $-98,65 \%$ & $466,85 \%$ \\
\hline \multirow{3}{*}{ تَّ } & 30 & $-19,71 \%$ & 4,65 & $1,32^{\mathrm{NS}}$ & $76,71 \%$ & $-100,00 \%$ & $299,32 \%$ \\
\hline & 60 & $-58,63 \%$ & 2,89 & $3,09^{\mathrm{NS}}$ & $93,57 \%$ & $-100,00 \%$ & $692,86 \%$ \\
\hline & 90 & $-87,84 \%$ & 1,48 & $4,49^{\mathrm{NS}}$ & $96,78 \%$ & $-100,00 \%$ & $735,18 \%$ \\
\hline \multicolumn{2}{|c|}{ VOI } & $8,02 \%$ & 5,94 & 0,03 & $51,53 \%$ & $-99,99 \%$ & $653,64 \%$ \\
\hline \multicolumn{2}{|c|}{ VOI-P } & $3,82 \%$ & 5,83 & 0,15 & $51,63 \%$ & $-99,89 \%$ & $570,16 \%$ \\
\hline
\end{tabular}

Fonte: Resultados da Pesquisa. (*) Coeficientes não estatisticamente significativos ao nível de 1\%; NS Coeficientes não significativos ao nível de 5\%; ERM é o erro relativo médio.

No tocante à análise da volatilidade histórica, o aumento da janela recursiva reduz os erros de apreçamento para a volatilidade VMA, e de modo geral, aumenta os erros para as demais volatilidades históricas. Com exceção da volatilidade $\mathrm{VCH}$, houve subavaliação das opções com o uso da volatilidade histórica. Quanto à utilização da volatilidade implícita, verifica-se uma superavaliação das opções, porém ao comparar com os resultados obtidos com a volatilidade histórica, houve uma melhora significativa no apreçamento, principalmente na volatilidade implícita ponderada (VOI-P).

Em termos estatísticos, o teste de Tukey, por meio da diferença absoluta entre a média dos preços teóricos e do valor dos prêmios praticados no mercado, indica que os resultados para volatilidade histórica VMA, VHL e GARCH foram superiores a diferença mínima significativa calculada pelo teste, demonstrando que as médias não são estatisticamente iguais ao nível de significância de $5 \%$, com exceção da volatilidade VMA com 90 dias de janela recursiva. Cabe destacar que no apreçamento com essas volatilidades (VMA, VHL e GAR$\mathrm{CH}$ ), uma grande parcela da amostra foi subavaliada, enquanto que com a volatilidade histórica $\mathrm{VCH}$ e as volatilidades implícitas houve um equilíbrio entre as opções subavaliadas e superavaliadas. Com base nos erros relativos médios observados e no teste de Tukey foi feita a escolha da janela recursiva adequada para cada metodologia de extração da volatilidade histórica: 90 
dias para VMA, 30 dias para VHL e GARCH e 45 dias para VCH, sendo assim, parte-se para a análise dos diferentes extratos da amostra utilizada.

Em seguida, partiu-se para a análise dos erros de apreçamento de cada ano do período selecionado (Tabela 2). Verifica-se que o aumento dos contratos de opção de venda sobre futuros de café arábica de 2003 para 2004 e de 2006 para 2007 foi seguido de uma redução da subavaliação nos modelos com volatilidade histórica. Em contrapartida, nos períodos em que houve redução dos contratos futuros houve piora dos resultados de apreçamento. Cabe destacar que 2005 se configura com uma exceção, sendo que os resultados dos modelos com volatilidade histórica VHL e GARCH apresentaram leve melhora dos resultados, enquanto que os modelos com volatilidade VMA e VCH apresentaram um aumento da superavaliação das opções. Enfim, desse modo verifica-se que os resultados dos modelos com a utilização da volatilidade histórica estão diretamente relacionados com o nível de contratos transacionados, ou seja, com a liquidez de mercado.

TABELA 2 - RESULTADOS DE APREÇAMENTO DAS OPÇÕES DE VENDA COM VOLATILIDADES HISTÓRICA E IMPLÍCITA PARA CADA ANO DO PERÍODO ANALISADO

\begin{tabular}{rrrrrrrr}
\hline & VMA & \multicolumn{1}{c}{ VHL } & \multicolumn{1}{c}{ VCH } & \multicolumn{1}{c}{ CH } & VOI & VOI-P & \multicolumn{1}{c}{ CN* } \\
\hline $\mathbf{2 0 0 3}$ & $-25.48 \%$ & $-53.13 \%$ & $-11.63 \%$ & $-57.49 \%$ & $9.13 \%$ & $6.31 \%$ & 14655.00 \\
$\mathbf{2 0 0 4}$ & $-13.34 \%$ & $-44.83 \%$ & $35.38 \%$ & $-35.84 \%$ & $10.33 \%$ & $6.78 \%$ & 24253.00 \\
$\mathbf{2 0 0 5}$ & $14.21 \%$ & $-23.11 \%$ & $48.03 \%$ & $-27.20 \%$ & $12.29 \%$ & $-0.40 \%$ & 11867.00 \\
$\mathbf{2 0 0 6}$ & $-27.03 \%$ & $-49.35 \%$ & $-7.01 \%$ & $-32.25 \%$ & $7.60 \%$ & $2.82 \%$ & 12632.00 \\
$\mathbf{2 0 0 7}$ & $-6.63 \%$ & $-48.19 \%$ & $19.40 \%$ & $-25.38 \%$ & $14.51 \%$ & $12.69 \%$ & 27211.00 \\
$\mathbf{2 0 0 8}$ & $-11.92 \%$ & $-48.61 \%$ & $6.48 \%$ & $22.30 \%$ & $-0.26 \%$ & $0.48 \%$ & 25471.00 \\
$\mathbf{2 0 0 9}$ & $-17.10 \%$ & $-49.58 \%$ & $-22.91 \%$ & $-27.91 \%$ & $0.20 \%$ & $-1.58 \%$ & 5423.00 \\
\hline
\end{tabular}

FONTE: Resultados da Pesquisa. (*) Contratos Negociados.

Quanto a volatilidade implícita no-dinheiro, obtida por meio do procedimento de Macbeth e Merville (1979), independente do ano analisado, os resultados foram sistematicamente melhores do que com o uso da volatilidade histórica. $\mathrm{Na}$ análise da volatilidade implícita ponderada, não houve grandes divergências da volatilidade implícita, com exceção dos anos de 2005 e 2006, períodos que apresentaram quedas significativas dos contratos de opções de venda negociados. Ou seja, nessas ocasiões a ponderação da volatilidade implícita foi útil para contornar o problema de liquidez, melhorando os resultados de apreçamento. 
TONIN, M. T. ; COELHO, A. B. Apreçamento sobre as opções de venda ...

TABELA 3 - RESULTADOS DE APREÇAMENTO DAS OPÇÕES DE COMPRA PARA DIFERENTES GRAUS DE MONEYNESS

\begin{tabular}{|c|c|c|c|c|c|c|c|c|c|c|}
\hline \multirow{2}{*}{ Volat. } & \multicolumn{2}{|c|}{ DITM } & \multicolumn{2}{|c|}{ ITM } & \multicolumn{2}{|c|}{ ATM } & \multicolumn{2}{|c|}{ OTM } & \multicolumn{2}{|c|}{ DOTM } \\
\hline & ERM & Dif. & ERM & Dif. & ERM & Dif. & ERM & Dif. & ERM & Dif. \\
\hline VMA & $-25,41 \%$ & $2,26^{*}$ & $-6,00 \%$ & $0,50^{*}$ & $-4,74 \%$ & 0,97 & $-29,12 \%$ & 1,15 & $-41,66 \%$ & $0,91^{*}$ \\
\hline VHL & $-62,69 \%$ & $3,20^{\mathrm{NS}}$ & $-45,69 \%$ & $2,12^{\mathrm{NS}}$ & $-31,47 \%$ & $2,85^{\mathrm{NS}}$ & $-49,15 \%$ & $1,96^{*}$ & $-76,99 \%$ & $1,61^{\mathrm{NS}}$ \\
\hline $\mathrm{VCH}$ & $31,62 \%$ & 0,85 & $21,89 \%$ & $0,54^{*}$ & $7,22 \%$ & 0,36 & $-8,51 \%$ & 0,47 & $-33,05 \%$ & $0,68^{*}$ \\
\hline GARCH & $-53,26 \%$ & $2,95^{\mathrm{NS}}$ & $-20,21 \%$ & $1,05^{\mathrm{NS}}$ & $-15,16 \%$ & $1,58^{*}$ & $-43,93 \%$ & 1,49 & $-82,85 \%$ & $1,81^{\mathrm{NS}}$ \\
\hline VOI & $-23,49 \%$ & $2,21^{*}$ & $9,98 \%$ & 0,05 & $7,89 \%$ & 0,03 & $6,14 \%$ & 0,03 & $14,18 \%$ & $1,37^{*}$ \\
\hline VOIP & $-31,95 \%$ & $2,45^{*}$ & $5,34 \%$ & 0,05 & $5,93 \%$ & 0,13 & $1,87 \%$ & 0,13 & $4,17 \%$ & $1,45^{*}$ \\
\hline$n$ & 25 & & & & 21 & & 175 & & 24 & \\
\hline
\end{tabular}

FONTE: Resultado da Pesquisa

* Coeficientes não estatisticamente significativos ao nível de 1\%. NS - Coeficientes não estatisticamente significativos ao nível de 5\%. ERM é o erro relativo médio e Dif. a diferença entre os prêmios observados e estimados pelos diferentes modelos de apreçamento.

Na análise do grau de moneyness (Tabela 3) verifica-se que as opções mais afastadas do-dinheiro (DOTM e DITM) foram as que apresentaram os maiores erros relativos médios. Desse modo, verifica-se o fato estilizado conhecido como "sorriso da volatilidade" no apreçamento de opções sobre futuros de café arábica. Com exceção das opções no-dinheiro (ATM), os melhores resultados na precificação foram obtidos com a utilização da volatilidade implícita, sendo que a ponderação dessa volatilidade melhorou ainda mais os resultados de precificação, com exceção das opções DITM. Em termos estatísticos, os modelos de apreçamento em que utilizou-se as volatilidades históricas VHL e GARCH, não se mostraram adequados, dados que apenas para as opções OTM ambos foram estatisticamente significativos. 
TONIN, M. T. ; COELHO, A. B. Apreçamento sobre as opções de venda ...

TABELA 4 - RESULTADOS DO APREÇAMENTO DAS OPÇÕES DE COMPRA PARA DIFERENTES VENCIMENTOS

\begin{tabular}{|c|c|c|c|c|c|c|c|c|c|c|}
\hline \multirow{2}{*}{ Volat. } & \multicolumn{2}{|c|}{$\begin{array}{l}\text { MAR } \\
\end{array}$} & \multicolumn{2}{|c|}{ MAI } & \multicolumn{2}{|c|}{ JUL } & \multicolumn{2}{|c|}{ SET } & \multicolumn{2}{|c|}{$\overline{\text { DEZ }}$} \\
\hline & ERM & Dif. & ERM & Dif. & ERM & Dif. & ERM & Dif. & ERM & Dif. \\
\hline VMA & $4,99 \%$ & 0,02 & $-15,14 \%$ & 0,47 & $-11,29 \%$ & 0,26 & $-18,52 \%$ & $1,42^{*}$ & $-5,92 \%$ & 0,32 \\
\hline VHL & $-28,09 \%$ & $1,78^{\mathrm{NS}}$ & $-30,13 \%$ & $1,35^{\mathrm{NS}}$ & $-46,15 \%$ & $1,33^{*}$ & $-50,37 \%$ & $3,32^{\mathrm{NS}}$ & $-40,07 \%$ & $1,63^{\mathrm{NS}}$ \\
\hline $\mathrm{VCH}$ & $46,64 \%$ & 0,35 & $7,23 \%$ & 0,23 & $4,50 \%$ & 0,18 & $-2,41 \%$ & 0,56 & $22,04 \%$ & 0,54 \\
\hline GARCH & $-34,23 \%$ & $1,89^{\mathrm{NS}}$ & $-9,85 \%$ & 0,52 & $-29,83 \%$ & $1,10^{*}$ & $-22,74 \%$ & $1,69^{\mathrm{NS}}$ & $-32,00 \%$ & $1,12^{\mathrm{NS}}$ \\
\hline VOI & $9,03 \%$ & 0,75 & $10,96 \%$ & 0,14 & $0,89 \%$ & 0,10 & $9,50 \%$ & 0,02 & $5,23 \%$ & 0,02 \\
\hline VOI-P & $7,83 \%$ & 0,77 & $1,38 \%$ & 0,14 & $0,42 \%$ & 0,68 & $3,95 \%$ & 0,01 & $3,24 \%$ & 0,07 \\
\hline 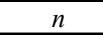 & & & 9 & & & & 42 & & 25 & \\
\hline
\end{tabular}

FONTE: Resultado da Pesquisa

* Coeficientes não estatisticamente significativos ao nível de 1\%. NS - Coeficientes não estatisticamente significativos ao nível de $5 \%$.

$\mathrm{Na}$ análise dos diferentes vencimentos do ativo-subjacente (Tabela 4) verifica-se que as volatilidades históricas VMA e VCH foram as que apresentaram os melhores resultados para os vencimentos de março e setembro, respectivamente, enquanto que para os demais contratos o melhor resultado foi da volatilidade implícita ponderada. Em termos estatísticos os modelos com volatilidade VHL e GARCH não se mostraram adequados, sendo que somente foram estaticamente significativos para os vencimentos de maio e julho, respectivamente. As volatilidades históricas VMA e VCH seguem a mesma dinâmica, sendo que do contrato de março para maio aumenta a subavaliação das opções, enquanto que do contrato de setembro para dezembro aumenta a superavaliação das mesmas. Quanto a ponderação da volatilidade implícita, houve melhora em todos os vencimentos analisados, sendo a melhora mais expressiva foi obtida no contrato de maio, um contrato com baixa liquidez, no comparativo com os demais contratos.

Enfim, busca-se avaliar os resultados de apreçamento considerando diferentes períodos de maturidade das opções (Tabela 5). Na análise dos modelos com volatilidade histórica, verifica-se que com exceção da volatilidade VCH há uma subavaliação das opções, independente do prazo de maturidade analisado. A volatilidade histórica VCH, assim com as volatilidades implícitas, superavalia as opções de venda. Com exceção das opções com prazos curtos de maturidades, os melhores resultados são obtidos com a utilização da volatilidade implícita ponderada. 
TONIN, M. T. ; COELHO, A. B. Apreçamento sobre as opções de venda ...

TABELA 5 - RESULTADOS DO APREÇAMENTO DAS OPÇÕES DE COMPRA PARA DIFERENTES MATURIDADES

\begin{tabular}{|c|c|c|c|c|c|c|c|c|c|c|c|c|c|}
\hline \multirow{2}{*}{$n$} & \multirow{2}{*}{ Time } & \multicolumn{2}{|c|}{ VMA } & \multicolumn{2}{|c|}{ VHL } & \multicolumn{2}{|c|}{ VCH } & \multicolumn{2}{|c|}{ GARCH } & \multicolumn{2}{|c|}{ VOI } & \multicolumn{2}{|c|}{ VOI-P } \\
\hline & & ERM & Dif. & ERM & Dif. & ERM & Dif. & ERM & Dif. & ERM & Dif. & ERM & Dif. \\
\hline 294 & $<45$ & $-16,34 \%$ & 0,53 & $-43,57 \%$ & $\begin{array}{l}1,3 \\
\text { NS }\end{array}$ & $3,60 \%$ & 0,12 & $-34,62 \%$ & 0,91 & $14,25 \%$ & 0,02 & $4,88 \%$ & 0,16 \\
\hline 357 & $45-90$ & $-8,39 \%$ & 0,51 & $-46,40 \%$ & 2,03 & $15,59 \%$ & 0,18 & $-20,37 \%$ & $\begin{array}{r}0,99 \\
\text { NS }\end{array}$ & $3,77 \%$ & 0,06 & $2,89 \%$ & 0,11 \\
\hline 298 & $>90$ & $-10,10 \%$ & 1,37 & $-42,42 \%$ & $\underset{\mathrm{NS}}{3,43}$ & $17,28 \%$ & 0,25 & $-23,90 \%$ & 2,13 & $6,98 \%$ & 0,01 & $3,91 \%$ & 0,17 \\
\hline
\end{tabular}

FONTE: Resultado da Pesquisa

* Coeficientes não estatisticamente significativos ao nível de 1\%. NS - Coeficientes não estatisticamente significativos ao nível de $5 \%$.

Com exceção dos procedimentos que incorporam na análise a volatilidade intradiária (VHL e VCH), verifica-se que, no comparativo entre os diferentes prazos de maturidade, as opções de venda com prazo médio de maturidade (45 a 90 dias) são melhor precificadas. Em termos estatísticos, a volatilidade VHL não foi estatisticamente significativa para nenhum dos prazos de maturidade utilizados, enquanto que a volatilidade GARCH só tem significância estatística para opções de até 45 dias de maturidade.

\section{Conclusão}

É reconhecida a importância histórica do café para a economia brasileira e com a crescente importância dos instrumentos de derivativos na gestão de risco do setor cafeeiro, o presente estudo buscou avaliar sob vários aspectos a aplicabilidade da Fórmula de Black no apreçamento de opções sobre contratos futuros de café arábica. Além da preocupação com a identificação da volatilidade mais apropriada a ser utilizada na determinação dos prêmios das opções agropecuárias, deu-se especial atenção aos resultados em diferentes extratos da amostra: diferentes anos, diferentes vencimentos, graus de moneyness e maturidade distintas.

De maneira geral, é visível o melhor desempenho do apreçamento de opções sobre futuros de café arábica com a utilização da volatilidade implícita do que as metodologias de cálculo com volatilidade histórica propostas. Considerando a ponderação da volatilidade implícita, verifica-se que essa metodologia é 
adequada para todos os modelos utilizados na análise das opções de venda, devido à redução dos erros de apreçamento.

Quanto à volatilidade histórica, uma dificuldade inicial é definir a janela recursiva a ser utilizada no cálculo. No presente estudo testou-se as janelas de 30, 45, 60,75 e 90 dias. Sendo que, uma janela recursiva maior melhora a apreçamento quando se utiliza a volatilidade histórica com o procedimento de média móvel (VMA). Porém, os procedimentos de Parkinson (VHL) e Garman e Klass (VCH) apresentam melhores resultados com janelas menores, de 30 e 45 dias, respectivamente.

Por meio da análise dos resultados em cada ano do período selecionado, buscou-se fazer um contraponto com a liquidez de mercado, comparando com o total de contratos negociados. Não é possível fazer uma correlação direta entre erros de apreçamento e número de contratos, porém, verifica-se que em períodos de aumento dos contratos negociados houve uma redução dos erros nos modelos com volatilidade VMA e VHC. Como grande parcela das observações é subprecificada nesses modelos, logo, houve uma melhoria no apreçamento em ambiente de maior liquidez.

No tocante ao conceito de liquidez, partiu-se para a análise de cada vencimento dos contratos futuros. Como o contrato de julho que apresenta a menor liquidez não foi o contrato com pior resultados, não foi possível estabelecer uma relação direta entre liquidez e erros de apreçamento. Porém, verificouse o aumento da subavaliação no comparativo com o vencimento de março para maio e aumento da superavaliação no comparativo do vencimento de setembro com dezembro, com a utilização das volatilidades históricas VMA e VCH. Ou seja, o aumento da superavaliação ou subavaliação está relacionado a rolagem de posição entre os contratos futuros em períodos primordiais do ciclo produtivo do café.

Na análise dos diferentes graus de moneyness, comprovou-se que nas opções sobre futuros de café arábica também existe o fato estilizado conhecido como sorriso da volatilidade, em que os erros de apreçamento são maiores em opções mais afastadas do dinheiro e menores nas opções mais próximas do dinheiro. Quanto ao período de maturidade, os resultados destoam dependendo do procedimento de cálculo da volatilidade adotada. As volatilidades que incorporam na análise a volatilidade intradiária (VHL e VCH), apresentam os melhores resultados para o menor período de maturidade analisado, enquanto que as demais volatilidades, o melhor resultado de apreçamento ocorre quando se precificam opções de vença com prazo médio de maturidade.

Enfim, dada a diversidade de modelos existentes para o apreçamento de opções sobre contratos futuros, a abordagem utilizada nesse trabalho, sob diversos aspectos para diversos extratos da amostra pode ser replicada em outros trabalhos para analisar os resultados de modelos mais complexos. Entre as contribuições desse trabalho, estão o procedimento de Macbeth e Merville 
(1979) para o cálculo da volatilidade implícita no dinheiro e a ponderação na volatilidade implícita que foi capaz de amenizar o problema da baixa liquidez na negociação de opções de futuros de café arábica, melhorando os resultados dos modelos de apreçamento. Porém, no que tange a volatilidade histórica, apesar das diversas técnicas existentes, há uma carência de metodologias que captem adequadamente a volatilidade intradiária, para empregá-la de maneira eficiente na precificação de opções.

\section{Referências}

AGUILAR, J. GARCH, Implied Volatilities and Implied Distributions: An Evaluation for Forecasting Purposes," Working Paper Series 88, Sveriges Riksbank (Central Bank of Sweden), 1999.

BANCO CENTRAL DO BRASIL. Taxa de Juros DI - 1 Dia. Disponível em: <www. bcb.gov.br>. Acesso em: 28/01/2009.

BARONE-ADESI, G.; WHALEY, R. Efficient analytical approximation of American option values. Journal of Finance, Vol. 42, No. 2, p. 301-320, 1987.

BARONE-ADESI, G. The Saga of American Put. Journal of Banking e Finance, n. 29 p. 2909-2918, 2005.

BJERKSUND, P.; STENSLAND, G. Closed-form approximation of American options, Scandinavian Journal of Management, Vol. 9, Suppl., p. 88-99, 1993.

BJERKSUND, P.; STENSLAND, G. Closed form valuation of American Options. Technical Report, Norwegian School of Economics and Business Administration, 2002.

BLACK, F. The pricing of commodity contracts. Journal of Financial Economics, $3,167-179,1976$.

BLACK, F; SCHOLES, M. The Pricing of Options and Corporate Liabilities. Journal of Political Economy, v. 81, n. 3, pp. 637 - 659, May/June, 1973

BOLSA DE MERCADORIAS, VALORES E FUTUROS - BM\&FBOVESPA. Relatório Agropecuário Mensal. Disponível em: <http://www.bmf.com.br/ portal/pages/imprensa1/ relatorios/relatoriosAgropecuarios.asp $>$. Acessado em: 05/01/2011.

BUSTOS, O. H. Outliers e Robustez. Revista Brasileira de Estatística, Rio de Janeiro, v. 50, n 193, p.1-129 jan/jun. 1989

CHALAMANDARIS, G.; MALLIARIS, A.G. Itô's Calculus and the Derivation of the Black-Scholes Option-Pricing Model. Handbook Of Quantitative Finance, 2008 Disponível em: <http://ssrn.com/abstract=1022386> Acesso em: $06 / 01 / 2009$.

COELHO, A. B.; PINHEIRO, S. C.; FERREIRA, F. V. A Fórmula de Black precifica corretamente as opções de compra sobre futuros agropecuários no Brasil? Uma aplicação para o caso do café arábica. Pesquisa \& Debate, São Paulo, n. 36, Nov. 2009.

EDERINGTON, L.H.; GUAN, W. “Why Are Those Options Smiling?” University of 
Oklahoma Center for Financial Studies Working Paper. July, 2000.

FARINA, E.M.M.Q.; ZYLBERSTAJN, D. Sistema Agroindustrial do Café. IN: INSTITUTO DE PESQUISA ECONÔMICA APLICADA (IPEA). Competitividade do Agribusiness Brasileiro. Relatório Final. Programa de Estudos dos Negócios do Sistema Agroindustrial (PENSA), Julho de 1998. 236 p.

FUTURE INDUSTRY ASSOCIATION. Trading Volume Statistics: Global Futures and Options Trading rose 25,6\% in 2010. March, 2011. Disponível em: <http:// www.futuresindustry.org/ volume-statistics.asp >. Acesso em: 06/04/2011.

GABE, J.; PORTUGAL, M. S.. Volatilidade Implícita Versus Volatilidade Estatística: Um exercício utilizando opções e ações da Telemar S.A. Revista Brasileira de Finanças, Rio de Janeiro, v. 2, n. 1, p. 47-73, 2004.

GENTIL JUNIOR, G. Sorriso da Volatilidade nas Opções de compra da Telemar PN usando dados intradiários. Dissertação (Mestrado de Administração) - Instituto COOPEAD de Administração da Universidade Federal do Rio de Janeiro, Rio de Janeiro, 2007.

HAUG, E. G. The Complete Guide to Option Pricing Formulas. New York: McGraw-Hill, 1997. 232 p.

HAUSER, S.; LAUTERBACH, B. The relative Performance of Five Alternative Warrant Pricing Models. Financial Analysts Journal, jan/fev. 1997

HOFFMANN, R. Estatística para Economistas. São Paulo: Pioneira Thomson Learning, 2006.

HULL, J.; WHITE, A. The pricing of options on assets with stochastic volatility. Journal of Finance, 42, 1987.

INSTITUTO DE ECONOMIA AGRÍCOLA - IEA. Café: Qual o Futuro? Disponível em: < www.iea.sp.gov.br > . Acesso em: 06/04/2010.

INTERNACIONAL COFFEE ORGANIZATION. Disponível em: <http//www. ioc. org $>$. Acesso em: 06/01/2009.

LEITE. D. R. S. Opções sobre contratos futuros de café na BM\&F: teste de modelos de precificação. Dissertação (Mestrado em Economia Aplicada) - Escola Superior de Agricultura Luiz de Queiroz, Universidade de São Paulo, São Paulo, 2000.

LEUTHOLD, R. M.; J.C.JUNKUS, J. C.; CORDIER, J. E. The theory and practice of futures markets. Lexington Books, 1989. $410 \mathrm{p}$.

LUCCAS, A. U. Modelo de Precificação de Opções com Saltos: Análise Econométrica do modelo de KOU no Mercado Acionário Brasileiro. Dissertação (Mestrado em Administração) - Faculdade de Economia, Administração e Contabilidade da Universidade de São Paulo, São Paulo, 2007. 189 p.

MACBETH, J.D.; MERVILLE, L. J. An Emperical Examination of the Black-Scholes Call Option Pricing Model. The Journal of Finance, n.5, December,1979

MALZ, Allan M. Do Implied Volatilities Provide Early Warning of Market Stress? RiskMetrics Journal. v.1, n.1, p.41-60, 2000.

MERTON, R. C. The Theory of rational option pricing. Bell Journal of Economics, v. 4, p. 141-183, 1973. 
MIKOSZEWKI, R. Precificação de opções de compra no mercado brasileiro: uma abordagem relativa de método numérico frente ao modelo de Black\& Scholes. Dissertação (Mestrado em Administração) - Universidade Federal do Paraná, Curitiba, 2003.

PARKINSON, M. The Extreme Value Method for Estimating the Variance of the Rate of Return. Journal Of Business, n. 53, p. 61-65, 1980.

PURCELL, W. D.; KOONTZ, S. R. Agricultural futures and options: principles and strategies. 2. ed. New Jersey: Prentice Hall, 1999.

RAMOS DA SILVA, T. J.; MACEDO, M. A. S. Opções sobre contratos futuros de café

arábica: uma avaliação da aplicabilidade do modelo de Black \& Scholes. In: Anais do $\mathbf{X}$

Simpósio de Engenharia de Produção (SIMPEP), Bauru, SP, 2003.

REINER, E.; RUBINSTEIN, M. Breaking Down the Barriers, Risk Magazine, vol.4, n.8, 1991

SANVICENTE, A. Z. ; MONTEIRO, R. C. . A guerra entre comprados e vendidos no mercado de opções de compra da Bolsa de Valores de São Paulo. Revista de Administração (USP), São Paulo, v. 40, n. 1, p. 34-43, 2005.

SILVA, M. A.; GUIMARÃES, B. V. Precificação de opções com volatilidade estocástica e saltos. Artigos Técnicos: Resenha 142, São Paulo: BM\&F, 1999, p.39-48.

STOLL, H. R., WHALEY, R. E. Hedging with futures contracts. Futures and options: theory and applications. Cincinnati: South-Western, 1993. p. 413.

VITIELLO, L. R. O cálculo da volatilidade implícita de opções de compra lançadas sobre contratos futuros. Resenha BM\&F, São Paulo, v. 138, p. 33-40, mar. 2000.

Recebido em: 13 de agosto de 2010

Primeira resposta em: 16 de novembro de 2010

Aceite em: 25 de abril de 2011 